\title{
Contextualisation of eLearning Systems in Higher Education Institutions
}

Link to publication record in Manchester Research Explorer

\section{Citation for published version (APA):}

Gwamba, G., Renken, J., Nampijja, D., Mayende, G., \& Muyinda, P. B. (2017). Contextualisation of eLearning Systems in Higher Education Institutions. In Proceedings of the 2017 International Conference on Interactive Mobile Communication, Technologies and Learning (IMCL2017) (pp. 327-338). (Advances in Intelligent Systems and Computing). Springer Nature.

\section{Published in:}

Proceedings of the 2017 International Conference on Interactive Mobile Communication, Technologies and Learning (IMCL2017)

\section{Citing this paper}

Please note that where the full-text provided on Manchester Research Explorer is the Author Accepted Manuscript or Proof version this may differ from the final Published version. If citing, it is advised that you check and use the publisher's definitive version.

\section{General rights}

Copyright and moral rights for the publications made accessible in the Research Explorer are retained by the authors and/or other copyright owners and it is a condition of accessing publications that users recognise and abide by the legal requirements associated with these rights.

\section{Takedown policy}

If you believe that this document breaches copyright please refer to the University of Manchester's Takedown Procedures [http://man.ac.uk/04Y6Bo] or contact uml.scholarlycommunications@manchester.ac.uk providing relevant details, so we can investigate your claim.

\section{OPEN ACCESS}




\title{
Contextualisation of eLearning Systems in Higher Education Institutions
}

\author{
Gerald Gwamba $^{1}$, Jaco Renken ${ }^{1}$, Dianah Nampijja ${ }^{2,3}$, Godfrey Mayende ${ }^{2,3, *}$ and Paul \\ Birevu Muyinda ${ }^{3}$ \\ 1. University of Manchester, UK \\ 2. University of Agder, Norway \\ 3. Makerere University, Uganda \\ * godfrey.mayende@uia.no
}

\begin{abstract}
The proliferation of digital technologies, and the emergence of global lifelong learning has steered the transformation of education from the predominant classroom based learning to more flexible technology enhanced learning. However, realizing technology enhanced learning's much anticipated benefits towards improving the educational potential of Higher Educational Institutions (HEIs) in Developing Countries (DCs) is still a challenge because of the high information systems failure rate. Research suggests potentially substantial misalignment between DC HEIs' E-Learning Management Systems (ELMS) and their institutional contexts. To explore this gap, this research employs a qualitative approach based on a case study of the Makerere University Electronic Learning Environment (MUELE). We employ the Aparicio eLearning systems theoretical framework to explore the extent to which DC HEIs are aligning their ELMS within their institutional contexts. Results indicate that, ELMS are not aligned to the context of DC HEIs implying that stringent measures need to be taken to close misalignment gaps.
\end{abstract}

Keywords: E-learning, Learning Management Systems, Higher Education Institutions, Developing Country Context

\section{$1 \quad$ Introduction}

The proliferation of educational and mobile technologies, and the emergence of global lifelong learning has steered the transformation of education. From predominant classroom based learning to independent technology enhanced learning. Research suggests that there has been significant growth in eLearning across all products and services globally. Mobile learning is among the fastest growing subsectors within the online learning mainstream because of the improved access to affordable mobile technologies and the affordances that it brings among African citizens.

The rapid proliferation of eLearning within Higher Educational Institutions (HEIs) has been a result of social-economic drivers such as increased competitiveness (Sekiwu \& Naluwemba, 2014), cost effectiveness and reach (Lee-post, 2009), emergence of a mobile citizenry (Hossein, 2015; Vaiva, Edita, Daiva, Vladislav \& Kathy, 2014),

adfa, p. 1, 2011 .

(C) Springer-Verlag Berlin Heidelberg 2011 
among others. In Uganda particularly, research suggests that government efforts to increase the literacy rates through Universal Primary Education (UPE) and Universal Secondary Education (USE) have spurred a significant increase in student enrolments. This has mounted pressure on educational institutions already limited by space and other resources. In a bid to meet this demand particularly in HEIs, the Ugandan government is encouraging investment in eLearning as a suitable alternative (Mayoka \& Kyeyune, 2012).

The emergence of digital mobile technologies has steered the incorporation of mobile devices, services and platforms into learning environments with increasing adaptation to suit the mobile delivery. This has propelled major changes in the design of today's Learning Management Systems (LMS) with mobile supported features allowing even further flexibility for eLearning delivery and accessibility.

However, realizing technology enhanced learning's much anticipated benefits towards improving the educational potential of HEIs in DCs is still a challenge. This is because most information system implementations fail in some way (Masiero S. 2016; Heeks 2003). Research suggests that there could exist substantial mismatches between the alignments of Developing Country (DC) HEIs' E-Learning Management Systems (ELMS) and their institutional contexts (Gwamba, Mayende, Isabwe, \& Muyinda, 2017).

Moreover, some research focused on the development of information systems in DCs reveals that most IS projects fail to realise their anticipated benefits, and majority are externally funded especially when project funding is exhausted (Ssekakubo et al. 2011; Kinyua 2015). As a corrective, there is need for eLearning policy frameworks among African countries. The policy could allow for shared eLearning experiences of others through government led efforts providing centrally coordinated eLearning strategies that align with national goals, educational reforms and technology. Proposed eLearning strategies thus should lay out a road map for the eLearning architecture, resolve curriculum issues, guide on capacity development, and management of systems, content development and infrastructure among others (Manji et al., 2015).

Similar strategies have further focused on developing well-structured ideal solutions that all players should follow to achieve more successful eLearning developments (Manji et al., 2015; Mtebe, 2013; Ssekakubo, Suleman, \& Marsden, 2011). This is however hard to achieve since the question of contextual alignment of eLearning takes precedence. To be specific, a one-size-fit-all approach is undesirable because of the variance in contextual factors. Some universities have introduced eLearning for administrative, management and registration context which is far from ELMS core educational functions. Given that institutions operate in different contexts, many factors shape eLearning development ranging from technology, to social and pedagogical aspects among others (Aparicio, Bacao, \& Oliveira, 2016). Moreover, whilst government led policies where educational opportunities are identifiable; institutional realities within DCs present unique challenges most of which have not yet been adequately addressed.

This research employs an exploratory approach seeking to better understand the influence of the social and technical contexts of HEIs in DCs on their LMS implementations. We thus seek to answer the research question: To what extent are DC HEIs' ELMS aligned within their institutional contexts? In this paper, we attempt to answer this 
question by using the holistic eLearning systems theoretical framework to analyse how the institutional context aligns with the LMS. In the next sections of this paper, we present the approaches and methods used in section 2, followed by research findings in section 3. Then discussions in section 4. We then draw conclusions of the outcomes and limitations of the research in Section 5.

\section{Research Approach and Methods}

\subsection{Research Framework}

Aparicio et al. (2016) proposed a holistic E-Learning Systems Theoretical Framework (E-LSTF) which classifies key inter-connected factors that operate within an eLearning system domain that could lead to "successful" eLearning systems development. These factors are characterised in terms of people, technology and services dimensions.

In the framework, people interact with the systems and the technologies facilitate the direct and indirect interactions of different stakeholder groups, and further enable integration of eLearning content and collaborative tools (Aparicio et al., 2016). The services on the other hand integrate all the activities related to both instructional strategies and pedagogical models used within learning spaces. Therefore, the eLearning system provides the environment for complex interactions between various dimensions. In analysing the E-LSTF, we will critically assess the development of the different dimensions to gauge the extent of alignment of DC HEIs' ELMSes within their institutional contexts. The institutional context may focus on the environment which is constituent of a system of geographical, political, social-cultural and other factors as described in Heeks (2006, p.4-5).

Whereas other models may tend to focus on the extremes of social or technical aspects - a socio-technical approach like the e-government analogy in Heeks (2006); the E-LSTF combines both the soft social and hard technical extremes. The socio-technical/hybrid balance spans not only the system design side but also the implementation side. Accordingly, striking a social-technical balance could drive further alignment of IS within the institutional environment. In this regard, models that explore the nature of environmental forces whilst mapping them on technical aspects could be more ideal in informing IS in DC institutions, which resonates with the E-LSTF. It is however worth acknowledging that the E-LSTF is a newly published theory (2016) and has thus not yet gained a lot of validation putting our case among those to first validate this theory. The E-LSTF is well suited in the contextual alignment of ELMSes in DC HEIs since its constructs are focused on people, technology and activities, all of which are dimensions of the hybrid IS perspective (Aparicio et al., 2016; Heeks, 2006). Investigating the extent of alignment of ELMS within the institutional context based on the proposed model seeks to pre-empt a discussion surrounding how well information systems fit within the micro/macro environment of DC HEIs. 


\subsection{Research methods}

Our study employed qualitative methods using guided interviews and observations. Interviews with 8 key respondents were based on a set of semi-structured questions derived from the E-LSTF conceptual framework; interviews lasted 30-45 minutes. All interviewed respondents were selected based on their positions as experienced eLearning practitioners at Makerere University. Data collection was further guided by themes including "context of DC HEI" and "LMS alignment" derived from the research question. We used the case study research strategy characterised with a rich background on IS adoption in a DC HEI to allow for further discussions. To freely allow respondents to further express themselves, emails questionnaires were in addition to face interviews sent to respondents. This further provided free expression especially on controversial topics. The interviews were transcribed, and inter-rater reliability was employed. Reference to institutional literature was vital in enhancing data triangulation. The data was analysed through thematic analysis based on ELMS system theoretical framework.

\subsection{The Development of ELMS in Makerere University}

Figure 1 shows the development of computer assisted learning dating back to 2001, ten (10) years since the inception of distance learning.



Fig. 1. Overview of the Development of ELMS at Makerere University 
Makerere University (MAK), through its present Institute of Open Distance and eLearning $(\mathrm{ODeL})$, introduced first generation distance learning which later developed to second generation mode as referred to by (Taylor, 2001). The university is gradually adopting the use of ICTs for teaching, learning, research and knowledge dissemination. In recognising the need to improve efficiency in service delivery for academic, library, financial and human resources, MAK embarked on a drive to integrate IS within its core business operations (Tusubira, Mulira, Kahiingi, \& Kivunike, 2008).

The eLearning environment at MAK is however characterised by poor student support systems. Justifiably, present eLearning is developed at a slow pace through adopting a blended approach to supplement the traditional face to face mode of instruction. According to Figure 1, the first ELMS was introduced around 2003 in response to the ICT Master plan 2002-2004 (Greenberg \& Versluis, 2005). With support from Tufts University, MAK later introduced Blackboard LMS in 2003 but due to financial constraints, the replacement with an open source LMS - KEWL - in 2006 provided a sustainable alternative (Tusubira et al., 2008). KEWL's limited stability and errors at the time saw MAK opt for a more stable choice of Moodle which is better suited to handle a larger student population estimated at more than 40,000 students as of 2013/14 academic year.

\section{$3 \quad$ Findings}

Our findings are presented following a detailed look at each of the ELSTF framework dimensions offering valuable aspects to inform the contextual map of ELMS within DC HEIs.

\section{1 eLearning Technologies}

Technology plays a vital role in the implementation of collaborative web-based learning systems and tools that enable students and teachers to access shared workspaces through flexible and persistent connectivity. The ELST Framework categorises technologies in terms of content, collaboration and communication.

Content Technologies. Taking a keen interest on content is paramount since it is what delivers the business benefits of eLearning and it is what adds the knowledge, skills and capabilities to people. Data gathered from MAK staff reveals that a diversity of content technologies are supported on MUELE. Accounts from 8 respondents points to the parallel use of both analogue and digital content technologies for supporting institutional programmes. From respondent accounts, IODeL is currently focusing on training university teachers to develop instructional content based on e-pedagogy designs and content restructuring as a mechanism for interactive instructional design. This is one of the primary aims of the currently running projects. 'Most of what people do is to upload content, majorly knowledge repositories because from my audit of the system, that's what I see people do' (said one respondent). This depicts that the use context 
of the system is mainly for data storage as opposed to a learning platform which contradicts the use of the system by design. Conversely, 2 of the 8 respondents revealed that they had used commercial content development tools beyond Moodle with enhanced content development capabilities not yet affordable to MAK. Overall, content technologies identified were knowledge repositories, assessments, documents, text based authoring tools and limited digital audio/video. Whereas the above list of content technologies is dominant in MUELE, their overall alignment within the institutional context largely remains volatile, and partly inclined to social and institutionalization aspects.

Communication Technologies. One of the core functionalities of ELMS is the ability to offer flexible communication across diverse groups. Contextually, MAK offers traditional face to face programmes by establishment which affects the choice of communication technology to integrate in MUELE. Ideally the most popular communication technologies acknowledged are knowledge discussion forums, social networks and email. Data gathered suggests that videoconferencing for example was not planned during the university's establishment of eLearning. Based on staff accounts, some respondents argued that other institutions like the University of Nambia preferred videoconferencing due to harsh climatic conditions which hampered frequent human mobilities. However, the misalignment could emanate from the fact that MAK did not plan for such technologies within its establishments. Interestingly, from some respondents, 'the distance learning centres that were established as extramural centres were later transformed into population catchment centres - taking the university to the people'. Whereas by design, videoconferencing capabilities do exist for Moodle, they are highly resource dependent and would not be an ideal option for MAK given its financial constraints (Schenkel, 2013; Ssekakubo et al., 2011; Tabaire \& Okao, 2010). For social networking, WhatsApp, Facebook and Twitter are outside the domain of MUELE. However, Moodle supports chat services with additional plugins for audio/video integration by design. These capabilities have not been utilised and according to some respondent accounts, Moodle inbuilt social networking tools have not been popularised, and rightly afforded within MUELE.

Collaborative Technologies. These offer unprecedented opportunities for environments that foster group related activities suitable for off campus learning geographically consolidating learners and teachers. Results indicate an individualized, and highly personal social cultural learning context with in eLearning at MAK. '.. the pedagogical culture within MAK is more inclined towards individualised competitive teaching/learning as opposed to collaboration' (said one respondent). Such sentiments and more work against the culture of ELMS deterring group cooperation, which deters collaboration within MUELE. Through collaborative learning technologies, institutions and educators are in a better position to learn from each other, encouraging resource sharing to more openness that consolidate multidisciplinary user groups (The NMC Horizon Report, 2017). Thus, sharing and learning in groups as opposed to individualised learning has the power to facilitate deeper learning to optimise the full versatility 
of the ELMS. Despite the availability of tools like forums, chats and multiuser areas like blogs that facilitate multidisciplinary sharing, evidence from 5 respondents indicates that the collaborative power of MUELE is yet to be utilized. Evidence of strong external collaborations beyond MUELE creates a competitive force emanating from social networking tools like Facebook, WhatsApp, among others. "Facebook, WhatsApp among other tools provide faster and more reliable connectivity compared to the platform characterised with frequent downtime," narrates respondent 7. Earlier research on major impediments on ICT developments in MAK confirms this sentiment (Mayoka \& Kyeyune, 2012; Opati, 2013; Ssekakubo et al., 2011). However, all 8 respondents affirm that a clear roll plan for institutionalising eLearning has not yet been agreed upon causing serious misalignment issues. MUELE, on the one hand, provides several collaborative technologies, which we now found to be largely unused. On the other hand, collaborative technologies outside of MUELE are used by the university showing that the ELMS offering does not align with the collaboration requirements of the institution.

\subsection{E-Learning Stakeholders}

According to the Aparacio et al (2016) ELMSF, stakeholders are all people within the wider institutional domain (internal and external) who make use of the IS including customers, suppliers, professional associations and board and others. Results indicate that students, academic and administrative staff are the principal stakeholders in MUELE. Whereas students and lectures are the main consumers, it would be pertinent for us to fully conceptualise the role that other groups play in shaping major ELMS change and development. However, the newly approved eLearning policy refers to eLearning stakeholders as "ODeL target clients" without clearly spelling out the scope of this domain but rather refers to other institutional policies (Makerere University, 2015). This could create wider confusion for future application given the fact that further reference is made on an open admission policy that cartels many diverse groups of real, ideal or potential end-users. 'The current ELMS efforts are primarily targeting students alone and the system has not yet envisioned other groups within the university's establishment as potential customers' (said one respondent).

In the supplier categorisation, lecturers (facilitators) and MUELE technical team (administrators) emerged as the main other sub categories. No interests were noted on the remaining categorisations of professional associations, SIGs and Board \& Shareholders. Exception however emerged when one respondent revealed the viability of donors as key stakeholders within the MUELE value chain. This argument certainly demonstrates the level of influence of donors in shaping major ELMS developments in DC echoed in earlier research findings (Faridha, 2005; Mayoka \& Kyeyune, 2012; Moucheraud et al., 2017; Opati, 2013; Ssekakubo et al., 2011; Tusubira et al., 2008). Some of these assertions depict how MUELE does not take on all stakeholders; yet a successful and sustainable eLearning system presuppose stakeholders' involvement. It 
is also equally important to recognise activities of all institutional stakeholders as vital within an LMS ecosystem to allow for LMS contextualization ${ }^{1}$.

\subsection{E-Learning Activities}

E-learning activities integrate all the services delivered by the ELMS. MUELE makes use of some pedagogical models to practicalise eLearning within the institution. A total of 7 respondents agreed that whereas MUELE offers collaborative learning groups, they have not engaged in any contributions online. However, results reveal that collaborative groups are open to both staff and students. Whereas MUELE offers this provision, the fact that none of the respondents was interested in participating in any collaborative group contributions for any reason demonstrates that adoption issues do exist for example negative user attitudes, poor user interfaces etc. Distributed learning is popularised in MAK although misalignments could be evidenced due to reliability issues. Results reveal that there exists limited participation in online knowledge building communities which resonates with earlier findings since MUELE is not institutionalised and current efforts to engage with students online are out of individual motivation alone. This lack of ELMS institutionalization is partially the cause of eLearning misalignments at MAK. MUELE has been customised to offer several instructional delivery strategies to supplement the face to face instructions. Only 1 respondent reported to have contextualised content to suit the course needs. All staff agreed that whereas there exist current efforts to contextualise courses to suit online delivery, many existing courses uploaded on the platform are not contextualised. And as such, the ELMS simply enable online replication of offline teaching and learning approaches.

\section{Discussions}

E-learning systems promote a culture of group based learning and thus could align well in societies that have a richer history of collaborative work principles. Our results indicated that it has been widely recognised that MAK community is more individualist as opposed to collectivist (Hofstede, Hofstede, \& Minkov, 2010) and this wider recognition cuts across the administrative, academic and student domains. Collaborative learning promotes mutual assistance and sharing (Mayende, Prinz, Isabwe, \& Muyinda, 2016), which is majorly not the reality in MAK given the high prevalence of individualised recognition from assessment, promotion and appraisal. Collaborative services like communities of practice are argued to offer wider opportunities for distance learners to connect with instructors and peers for constant feedback about a shared question or subject matter (The NMC Horizon Report, 2017). Whilst some respondent showed use of other collaborative tools outside MUELE, issues of co-authorship and/or codevelopment of materials, reports and projects between different stakeholders were not well integrated; yet they are the backbone to collaborative eLearning systems. One respondent had this to say ... "we are dealing with people in the same locality. eLearning

\footnotetext{
${ }^{1}$ Contexualisation means using electronic learning management system, adding and replacing rich and interactive media content, and use of hyperlinks for non-linear navigation that befits individual courses.
} 
is mainly for supporting face to face learners, yet we meet physically. Why go through the hustle of troublesome net that is off sometimes". This indicates mixed reactions on staff attitudes for supporting eLearning. Kisanga \& Ireson (2015) acknowledges that formal stakeholder training in basic and professional ICT skills encourages a positive change in perception and attitude minimising resistance to change. Thus, misalignments could stem from the fact that while as MAK has deliberately decided to evolve its pedagogy to embrace collaborative learning activities, evidence from the ELMS analysis shows that this pedagogy has not yet diffused. The current eLearning system has not yet succeeded in enabling the pedagogical change and we therefore consider this an institutional misalignment. Other factors technological factors like limited internet could explain the limited use.

Beyond this, past institutional planning in comparison with current emerging eLearning developments could have taken the institution by surprise. MAK was originally founded as a technical school, and grew into a university with extramural centres. Results further revealed that MAK later opened regional population catchment centres for offering brick and mortar learning programmes in Lira, Jinja and Fort Portal. Since eLearning could shift the institution's traditional instructional mode, this could adversely affect the institution's rich traditional learning heritage and identity. The current blended approach at MAK provided a better contextual fit and alignment of ELMS within the existing traditional instructional framework.

The proliferation of other simpler collaborative technologies and the emergence of digital social networking tools have adversely affected the usability of MUELE. Further evidence beyond staff accounts suggests that many students spend a considerable amount of time on social media and enjoy more collaborative learning beyond MUELE (Opati, 2013). Moreover, the portability, reliability and mobile accessibility of competing social media platforms like Facebook and WhatsApp perhaps makes them preferable to stakeholders.

The wider view of LMSes as repositories or archiving systems for educational materials by most stakeholders should not be down played. This wider application resonates well with the staff selection of knowledge repositories and content upload among the most popular content technologies. ELMSes as knowledge repositories would centralise educational content from various suppliers both internal and external to the institution for easy access. However, the lessening convergence of LMS to mere repositories of materials would render ELMSes library systems as opposed to learning platforms which contradicts the definition of LMSes (Oxagile, 2016). On the other hand, absence of an institutionlised policy to guide the rollout of the ELMS at MAK could suggest that MUELE is still in its infancy. Relatedly, "the current contestation of the IODeL policy by the MAK management is still far from resolution," (said a respondent). This lack of an operational institution policy is a major impediment to eLearning contexualsiation at MAK. MUELE like other information systems LMS has the power to integrate with other systems in what could be referred to as Enterprise Resource planning (ERP) creating a unified environment for centralised access and share point. This functionality is not yet utilised. 'One problem that we have is that MUELE and other systems within the university fraternity are not connected or integrated together' said a respondent. Integrating information systems has the potential to facilitate faster 
access and sharing of institutional resources, and to minimise data storage costs. Despite MAK's efforts to establish a road map to guide ICT institutionalisation, ELMS alignment within the university context remains a serious challenge. This is further reinforced by the fact that most ELMS development efforts are centered on satisfying donor interests as opposed to the larger institutional objectives. As such, the context of MAK may not be significantly different from other institutions in DCs (Ssekakubo et al., 2011) with similar conditions.

\section{Conclusions}

Our research revealed that DC HEIs have unique contexts as compared to developed countries from where ELMSes originate. DC contexts must put in consideration further continuity of ELMS initiatives beyond initial support. Contextual alignment of ELMS could be better referred to as a "hybrid" approach since it combines the extremes of social and technical aspects as a consolidation of "contextual aspects" and "eLearning systems dimensions". Outcomes indicate that to a certain extent, ELMSes are aligned to the context of DC HEIs in the following ways:

- The establishment of eLearning as an informal approach to supplementing the face to face education could account for its current development. eLearning has not yet been institutionalised, since there exist gaps in ELMS rollout.

- Elearning emanates from external funding whose target is primarily to fulfill donor objectives with the university only partially realising targets.

However, to a certain extent, DC HEI's ELMS are not aligned with their institutional contexts:

- There is a misalignment between culture and the ELMS design requirements. Our evidence highlighted this in terms of learning collaboration.

- Institutional establishment is misaligned with the strategic planning for eLearning.

- Resources disparities evidenced in MAK work against ELMS development and continuity.

- Misalignment in management support and strategic planning.

- Misalignment in stakeholder competences in content development, and ePedagogies.

Conclusively, MUELE is to a considerable extent, not aligned with the context of Makerere University. This may equally apply to other DC HEIs within similar contexts. This implies that ad-hoc actions need to be taken to close the misalignment gaps e.g. through hybrid approaches to assure sustainability and realization of potential benefits that can be derived from eLearning. Suggested approaches may include; practicalising ELMS institutionalisation, management commitment (for ownership, resource provision and leadership oversight), strengthening collaborative workplaces, ELMS strategic planning and forecasting, ELMS specific capacity development among others. In summary, our research is meant to raise awareness on the organisational environment in DC HEIs and analyse how foreign digital technologies align within DC institutions. Finally, our research reveals that, the organisational environment plays a significant 
role in shaping the alignment of information systems in general as evidenced from research results in resonance with the framework.

\section{Acknowledgement}

The work reported in this paper was financed by Equity \& Merit Scholarship and the DELP project which is funded by NORAD. Acknowledgements also go to the University of Agder and Makerere University who are in research partnership.

\section{$7 \quad$ References}

Aparicio, M., Bacao, F., \& Oliveira, T. (2016). An e-learning theoretical framework. Educational Technology and Society, 19(1), 292-307.

Docebo Report. (2014). E-Learning market trends and forecast: 2014 - 2016 Report. A report by Docebo. Retrieved from https://www.docebo.com/landing/contactform/elearning-market-trends-andforecast-2014-2016-docebo-report.pdf

Faridha, M. (2005). Towards Enhancing Learning with Information and Communication Technology in Universities; A Framework for Adaptation of Online Learning. A Research Dissertation Submitted to the Graduate School in Partial Fulfillment of the Requirements for the award of t. Makerere University.

Greenberg, A., \& Versluis, G. (2005). Sida Supported ICT Project at Makerere University in Uganda. Evaluation. Retrieved from http://www.sida.se/contentassets/48d6c85ee2b845fca035baa5758983ab/sidasupported-ict-project-at-makerere-university-in-uganda_2094.pdf

Gwamba, G., Mayende, G., Isabwe, G. M. N., \& Muyinda, P. B. (2017). Conceptualising Design of Learning Management Systems to address Institutional Realities. Paper presented at the International Conference on Interactive Collaborative Learning.

Heeks, R., 2003. Success and Failure Rates of eGovernment in Developing/Transitional Countries, eGov4Dev.

Heeks, R. (2006). Implementing and managing eGovernment an international text. SAGE.

Hofstede, G., Hofstede, G. J., \& Minkov, M. (2010). Cultures and Organizations: Software of the Mind. Revised and Expanded. New York: McGraw-Hill USA (3rd ed.). Retrieved from http://geert-hofstede.com/books.html

Hossein, M. (2015). Computers in Human Behavior: Investigating users' perspectives on e-learning: An integration of TAM and IS success model.

Kinyua, A.W., 2015. Design reality gap framework for post implementation evaluation of the national ict policy., (July).

Lee-post, A. (2009). e-Learning Success Model: an Information Systems Perspective, $7(1), 61-70$.

Manji, F., Jal, E., Badisang, B., \& Opoku-mensah, A. (2015). The Trajectory of change:Next steps for education. eLearning Africa Report. 
Masiero S., 2016. The origins of failure: seeking the causes of design-reality gaps. Information Technology for Development, 22(3), 487-502.

Mayende, G., Prinz, A., Isabwe, G. M. N., \& Muyinda, P. B. (2016). Learning Groups for MOOCs: Lessons for Online Learning in Higher Education. Paper presented at the 19th International Conference on Interactive Collaborative Learning (ICL2016), 21-23 September, Clayton Hotel, Belfast, UK.

Mayoka, K., \& Kyeyune, R. (2012). An Analysis of E-learning Information System Adoption in Ugandan Universities: Case of Makerere University Business School. Information Technology Research Journal, 2(1).

Moucheraud, C., Schwitters, A., Boudreaux, C., Giles, D., Kilmarx, P. H., Ntolo, N., ... Bossert, T. J. (2017). Sustainability of health information systems: a threecountry qualitative study in southern Africa. BMC Health Services Research, 17, 1-12. https://doi.org/10.1186/s12913-016-1971-8

Mtebe, S. J. (2013). Making Learning Management System Success for Blended Learning in Higher Education in sub-Saharan Africa.

Opati, O. D. (2013). The Use of ICT in Teaching and Learning at Makerere University The Case of College of Education and External Studies. University of Oslo, 102, 37 ,.

Oxagile. (2016). Hostory and Trends of Learning Management System. Retrieved from http://www.oxagile.com/wp-content/uploads/2016/04/LMS-history01.png

Schenkel, M. (2013). The malaise of Makerere: underfunding, overcrowding. Retrieved from http://gga.org/stories/editions/aif-15-off-the-mark/the-malaise-ofmakerere-underfunding-overcrowding-strikes

Sekiwu, D., \& Naluwemba, F. (2014). E-learning for University Effectiveness in the Developing World. Global Journal of Human-Social Science ..., 14(3). Retrieved from http://www.socialscienceresearch.org/index.php/GJHSS/article/view/1162

Ssekakubo, G., Suleman, H. \& Marsden, G., 2011. Issues of Adoption: Have ELearning Management Systems Fulfilled their Potential in Developing Countries? , p.236,

Tabaire, B., \& Okao, J. (2010). Reviving Makerere University to a Leading Institution for Academic Excellence in Africa Synthesis Report of the Proceedings of The 3 rd State of the Nation Platform. Retrieved from http://www.acodeu.org/Files/Publications/PDS_8.pdf

Taylor, J. C. (2001). Fifth Generation Distance Education (Higher Education Series No. 40) - taylor01.pdf. Retrieved from http://www.c31.unioldenburg.de/cde/media/readings/taylor01.pdf

The NMC Horizon Report. (2017). NMC Horizon Report Preview 2017 Higher Education Edition. Retrieved from http://cdn.nmc.org/media/2017-nmc-horizonreport-he-preview.pdf

Tusubira, F. ., Mulira, N. K., Kahiingi, E. K., \& Kivunike, F. . (2008). Transforming Institutions Through Information and Communication Technology: The Makerere University experience. Intersoft Business Services.

Vaiva, Z., Edita, B., Daiva, V.-A., Vladislav, V. F., \& Kathy, K.-P. (2014). E-Learning as a Socio-Cultural System: A Multidimensional Analysis (p. 259,). 